\title{
AN EQUIVALENT CIRCUIT MODEL AND POWER CALCULATIONS FOR THE APS SPX CRAB CAVITIES
}

\author{
T. Berenc 1/26/2011
}

\section{Abstract}

An equivalent parallel resistor-inductor-capacitor (RLC) circuit with beam loading for a polarized $\mathrm{TM}_{110}$ dipole-mode cavity is developed and minimum radiofrequency (rf) generator requirements are calculated for the Advanced Photon Source (APS) short-pulse x-ray (SPX) superconducting rf (SRF) crab cavities.

\section{INTRODUCTION}

The polarized $\mathrm{TM}_{110}$ dipole-mode loss parameter is defined as [1]:

$$
k_{\perp}(y)=\frac{U_{\text {loss }}}{q^{2}}=\frac{\omega_{r} R^{(1)}(y)}{2 Q}=\frac{\left|V_{Z}(y)\right|^{2}}{4 U},
$$

where $R^{(1)}(y)$ is the shunt resistance of the dipole-mode transverse wake impedance, $U_{\text {loss }}$ is the energy lost to the dipole-mode by charge $q$ with vertical offset $y, Q$ is the loaded quality factor of the cavity, and $\omega_{r}$ is the cavity resonant frequency. $V_{Z}$ is the longitudinal voltage across the cavity gap with cavity stored energy, $U . \quad V_{Z}$ is dependent upon vertical offset, $y$, according to

$$
V_{Z}(y)=V_{m} \cdot y,
$$

where $V_{m}$ is in general a complex constant that allows the use of phasor notation in which the time varying longitudinal voltage can be written as $\operatorname{Re}\left\{V_{m} y \cdot e^{+j \omega t}\right\}$. The orientation of the fields and coordinate system are as shown in Fig. 1.

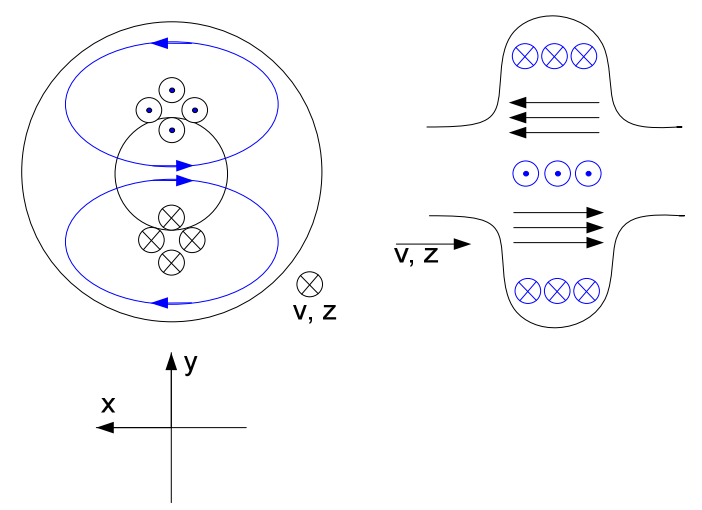

Fig. 1. Polarized $\mathrm{TM}_{110}$ mode fields [magnetic (blue), electric (black)] for an electron traversing the cavity gap in the $+\mathrm{z}$ direction receiving a kick in the $+\mathrm{y}$ direction.

The transverse voltage vector is related to the transverse gradient of the longitudinal voltage [1] by

$$
\vec{V}_{\perp}=\frac{j}{\kappa_{o}} \nabla_{t} V_{Z}
$$

Thus, the polarized mode transverse voltage vector is found to be independent of the vertical offset with a pure vertical component given by

$$
V_{\perp y}=j \frac{V_{m}}{\kappa_{o}}=-j V_{t},
$$

where $j=\sqrt{-1}, \quad \kappa_{o}=\frac{\omega_{r}}{c}$ is the wavenumber, and $V_{t} \equiv-V_{m} / \kappa_{o}$. The vertical kick imparted to a charge crossing the gap at time $t$ is given as

$$
y^{\prime}(t)=\operatorname{Re}\left\{\frac{q V_{\perp y}}{E} \cdot e^{+j \omega t}\right\}=\operatorname{Re}\left\{j \frac{-q V_{t}}{E} \cdot e^{+j \omega t}\right\}
$$

where $q$ is the charge with energy $E$ and the use of the negative sign in the definition of $V_{t}$ is for convenience in working with $e^{-}$charge, as will be appreciated later. Note that $\vec{V}_{\perp}$ is of the opposite sign of Ref. [1] because here we are assuming a harmonic oscillation of the form $e^{+j \omega t}$, whereas Ref. [1] assumes an oscillation of the form $e^{-j \omega t}$. In both cases Eq. 4 means that the magnetic field as shown in Fig. 1 leads the electric field as shown by 90deg.

As in [1], an $R / Q$, which is useful for an equivalent circuit model for the dipole-mode, can be defined as:

$$
\left(\frac{R}{Q}\right)^{\prime}=\frac{R^{(1)}(y)}{Q} \frac{1}{\left(\kappa_{o} y\right)^{2}}=\frac{\left|V_{t}\right|^{2}}{2 \omega_{r} U},
$$

which is independent of the radius and is a circuit definition that includes the factor of 2 in the denominator. The prime is used to be consistent with the notation of [2]. The cavity unloaded quality factor, $Q_{o}$, is defined as:

$$
Q_{o}=\frac{\omega_{r} U}{P_{c a v}},
$$

where the power dissipated in the cavity walls is given as

$$
P_{\text {cav }}=\frac{1}{2} \frac{\left|V_{t}\right|^{2}}{\left(\frac{R}{Q}\right)^{\prime} \cdot Q_{o}} .
$$

\section{EQUIVALENT CIRCUIT MODEL}

Using the relationship $V_{t}=V_{m} / \kappa_{o}$, the loss parameter of Eq. 1 can be written in terms of the transverse voltage as

$$
k_{\perp}(y)=\frac{\left|V_{t}\right|^{2}\left(\kappa_{o} y\right)^{2}}{4 U} .
$$

If an equivalent RLC circuit [3-4] is used to model the dipole-mode, then the energy $U$ can be expressed in terms of the equivalent circuit parameters as: 


$$
U=\frac{1}{2} C\left|V_{t}\right|^{2}
$$

$$
\text { where } \quad C=\frac{1}{\omega_{r}\left(\frac{R}{Q}\right)^{\prime}}
$$

represents the capacitance of the equivalent circuit. Thus, the loss parameter can be rewritten as

$$
k_{\perp}(y)=\frac{1}{2 C}\left(\kappa_{o} y\right)^{2}=\frac{1}{2} \omega_{r}\left(\frac{R}{Q}\right)^{\prime}\left(\kappa_{o} y\right)^{2} .
$$

\section{Point Charge Beam Loading}

From the fundamental theorem of beam loading [5, Sect. 6.1], as a charge $q$ crosses the cavity gap of Fig. 1 from left to right with vertical offset $y$, it will deposit energy equal to:

$$
U_{\text {loss }}=q^{2} k_{\perp}(y)
$$

The longitudinal beam-induced voltage is given as

$$
V_{Z b 0}=-2 q \cdot k_{\perp}(y)=-q \cdot \omega_{r}\left(\frac{R}{Q}\right)^{\prime}\left(\kappa_{o} y\right)^{2} .
$$

From Eq. 2 and Eq. $4 V_{t b 0}=-V_{Z b 0} /\left(\kappa_{o} y\right)$, giving a corresponding beam-induced transverse voltage of

$$
V_{t b 0}=\frac{q \cdot \kappa_{o} y}{C}=q \cdot \omega_{r}\left(\frac{R}{Q}\right)^{\prime} \kappa_{o} y \cdot
$$

The constant $V_{m}$ associated with the beam-induced voltage is

$$
V_{m b 0}=V_{Z b 0} / y=-q \cdot \omega_{r}\left(\frac{R}{Q}\right)^{\prime} \kappa_{o}^{2} y .
$$

The beam-induced longitudinal voltage has a direction that maximally opposes the motion of the inducing charge. Thus, it has the same direction whether it is above or below the cavity center, as depicted in Fig. 2 for an electron beam. However, the corresponding beaminduced $\mathrm{TM}_{110}$ mode fields, given by $\mathrm{V}_{\mathrm{m}}$ and $\mathrm{V}_{\mathrm{t}}$, change sign depending upon whether the inducing charge travels above, $y>0$, or below, $y<0$, the cavity center. The negative sign used in Eq. 13 and 15 has assumed that the charge crosses the gap at time $\mathrm{t}=0$.
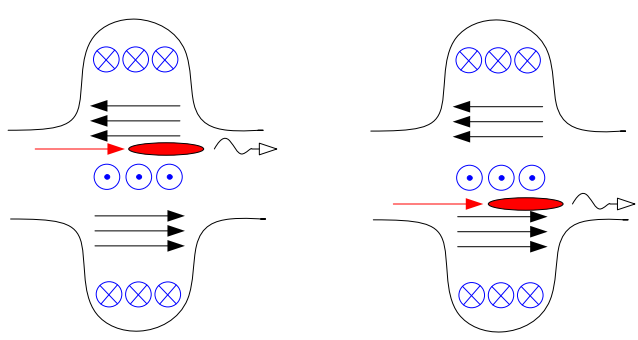

Figure 2: Depiction of an electron beam-induced electric field (red) in relation to the $\mathrm{TM}_{110}$ fields of Fig. 1.

\section{Single-Bunch Beam Loading}

Equation 13 gives the transverse voltage induced by a point charge. In general a bunch passing the cavity gap has a charge distribution. As in Ref. [5, Sect. 2.3], differential superposition can be used to determine the voltage induced by a bunch. Due to the offset dependent beam-loading, it is necessary to consider bunch tilt in the charge distribution. Ref. [6] uses a simple 2-macro particle model for bunch tilt while Ref. [7] employs a tilted Gaussian line distribution. The model of Ref. [7] results in a bunch-induced transverse voltage given by (see Appendix A)

$$
V_{t B 0}=q \cdot e^{\frac{-\left(\omega_{o} \sigma_{t}\right)^{2}}{2}} \kappa_{o} \omega_{r}\left(\frac{R}{Q}\right)^{\prime}\left[y+j \theta_{y z} \omega_{o} c \sigma_{t}^{2}\right] \cdot e^{-j \omega_{o} t_{o}}
$$

where $\omega_{o}$ is the operating rf radian frequency, $\sigma_{t}$ is the rms bunch length in sec, $y$ is the centroid vertical offset in meters, $\theta_{y z}$ is the bunch tilt in the $y$-z plane in radians, $c$ is the speed of light in $\mathrm{m} / \mathrm{sec}$, and $t_{o}$ is the time at which the centroid crosses the cavity gap. From Eq. 16 it is seen that bunch tilt adds a beam-loading component that is in quadrature with the centroid offset. The term $e^{-j \omega_{0} t_{o}}$ represents the phase shift that accounts for the bunch arrival time $t_{o}$.

\section{Multi-Bunch Beam Loading}

The methods of Ref. [5 Sect. 6.4] and Ref. [8] can now be invoked to determine the induced voltage from an infinite bunch train, $V_{t B}$, which can be expressed as

$$
V_{t B}=V_{t B 0} \cdot\left(\frac{1}{1-e^{-\delta_{o}(\beta+1)} e^{j \delta_{o}(\beta+1) \tan \phi_{z}}}-\frac{1}{2}\right),
$$

where $V_{t B 0}$ is the single bunch induced voltage given by Eq. 16, $\delta_{o}=\frac{T_{B}}{T_{o}}$ is the ratio of the bunch spacing $T_{B}$ to the unloaded cavity voltage decay time $T_{o}=\frac{2 Q_{o}}{\omega_{r}}, \beta=\frac{Q_{o}}{Q_{e x t}}$ is the coupling coefficient of the input coupler that is coupled to the cavity with external quality factor $Q_{e x t}$, and $\phi_{Z}$ is the cavity detuning angle given by $\tan \phi_{Z} \cong 2 Q_{L} \frac{\omega_{r}-\omega_{o}}{\omega_{r}}$. When $T_{B}$ is small compared to loaded cavity voltage decay time, $T_{L} \equiv \frac{2 Q_{L}}{\omega_{r}}=\frac{2 Q_{o}}{(\beta+1) \omega_{r}}$ with $Q_{L}$ the loaded quality factor, then $\delta_{o}(\beta+1) \cong 0$ and only the first term of Eq. 17 is significant. $V_{t B}$ can then be approximated as:

$$
\begin{aligned}
& V_{t B} \cong \frac{V_{t B 0}}{1-\left[1-\delta_{o}(\beta+1)\right] \cdot\left[1+j \delta_{o}(\beta+1) \tan \phi_{Z}\right]} \\
& \cong \frac{V_{t B 0}}{\delta_{o}(\beta+1)} \frac{1}{1-j \tan \phi_{Z}}=\frac{V_{t B 0}}{T_{B}} \frac{2 Q_{o}}{\omega_{r}} \frac{1 /(\beta+1)}{1-j \tan \phi_{Z}} .
\end{aligned}
$$


Substituting Eq. 16 for $V_{t B 0}$ in Eq. 18 gives:

$$
V_{t B} \cong-2 I_{D C} \cdot e^{\frac{-\left(\omega_{o} \sigma_{t}\right)^{2}}{2}} \kappa_{o}\left[y+j \theta_{y z} \omega_{o} c \sigma_{t}^{2}\right] \cdot e^{-j \omega_{o} t_{o}} \frac{R /(\beta+1)}{1-j \tan \phi_{Z}}
$$

where $I_{D C}=\frac{-q}{T_{b}}$ is the DC beam current with the negative sign used for convenience with $e^{-}$charge and $R=\left(\frac{R}{Q}\right)^{\prime} Q_{o}$ represents the equivalent circuit shunt impedance of the unloaded cavity. The term $\frac{R /(\beta+1)}{1-j \tan \phi_{z}}$ is recognized as the impedance of an equivalent circuit representation of the cavity with an input coupler (see Appendix B). The term $2 I_{D C} e^{\frac{-\left(\omega_{o} \sigma_{t}\right)^{2}}{2}} \equiv i_{o}$ is recognized as the rf component of the current of a Gaussian bunch train with $e^{\frac{-\left(\omega_{0} \sigma_{t}\right)^{2}}{2}}$ representing the bunch form factor Ref. [5, Sect. 3.3].

Equation 19 shows that for $\delta_{o}(\beta+1) \cong 0$ the beam-cavity interaction of the polarized $\mathrm{TM}_{110}$ dipole-mode can be modeled by the equivalent circuit shown in Fig. 3, where the equivalent beam loading current phasor is equal to

$$
\hat{I}_{B}=2 I_{D C} \cdot e^{\frac{-\left(\omega_{o} \sigma_{t}\right)^{2}}{2}} \kappa_{o}\left[y+j \theta_{y z} \omega_{o} c \sigma_{t}^{2}\right] \cdot e^{-j\left(\pi+\phi_{s}\right)}
$$

where the negative sign of Eq. 19 was replaced by $e^{-j \pi}$ and $\phi_{s} \equiv \omega_{o} t_{o}$ is defined as the "synchronous" phase angle of the beam.

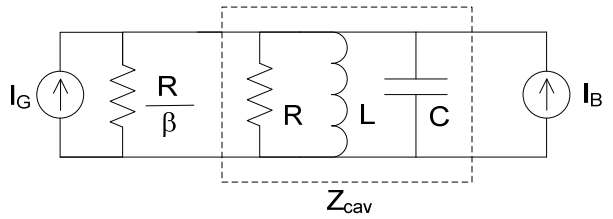

Fig. 3. Equivalent circuit model for the dipole-mode with multi-bunch passage beam loading.

The APS storage ring (SR) harmonic number is $\mathrm{h}=1296$ with a main rf accelerating frequency of $352 \mathrm{MHz}$. The SPX crab cavities will operate at $2815 \mathrm{MHz}$ (the $8^{\text {th }}$ harmonic of the main rf) and are expected to be operated with a loaded $Q$ value of $Q_{L} \cong 2 \cdot 10^{6}$ corresponding to $T_{L} \cong 226$ usec. For the equally spaced 24-bunch fill pattern envisioned for SPX, $T_{B} \cong 0.15$ usec giving $\delta_{o}(\beta+1) \cong 0.0007 \cong 0$.

The beam current can be written with the following parameterization to aid with calculations

$$
\hat{I}_{B}=\left|\hat{I}_{B}\right| \cdot e^{-j\left(\pi+\phi_{s}-\phi_{\theta}\right)}=\left|\hat{I}_{B}\right| \cdot e^{j \phi_{B}}
$$

with phase angle $\quad \phi_{B}=-\left(\pi+\phi_{s}-\phi_{\theta}\right)$

where $\left|\hat{I}_{B}\right|=2 I_{D C} \cdot e^{\frac{-\left(\omega_{o} \sigma_{t}\right)^{2}}{2}} \kappa_{o} \cdot\left|y+j \theta_{y z} \omega_{o} c \sigma_{t}^{2}\right|$

and

$$
\phi_{\theta}=\angle\left(y+j \theta_{y z} \omega_{o} c \sigma_{t}^{2}\right)
$$

represents the additional phase angle term introduced by bunch tilt in combination with beam offset.

The magnitude and phase of the equivalent beam loading current as a function of beam offset and tilt is shown in Figs. 4 and 5 for $\omega_{o}=2 \pi \cdot 2815 \mathrm{MHz}, \sigma_{t}=40 \mathrm{psec}$, and $I_{D C}=200 \mathrm{~mA}$. With these parameters, from Eq. 26, a pure beam tilt of $\sim 0.117 \mathrm{rad}$ (6.76 deg) has the same magnitude as a pure beam offset of $1 \mathrm{~mm}$.

$I_{B} \mid$ vs Beam Tilt $(y>0)$

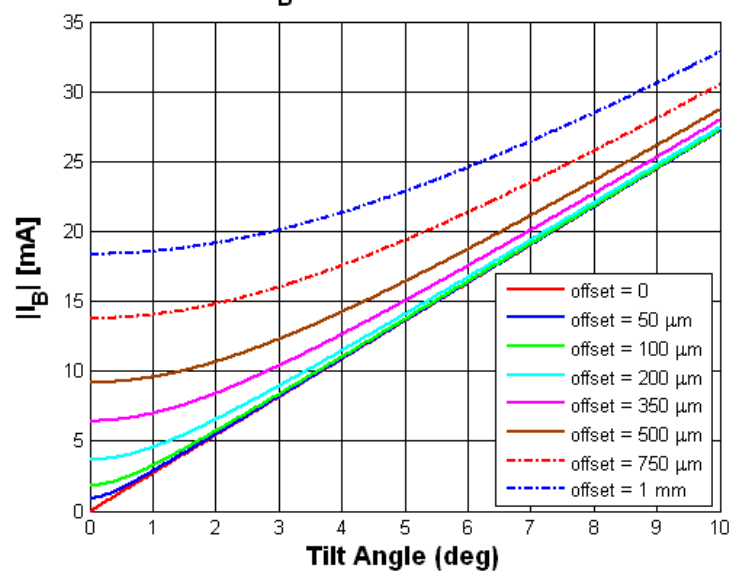

Fig. 4. Equivalent beam-loading current magnitude vs. beam tilt for various beam offsets.

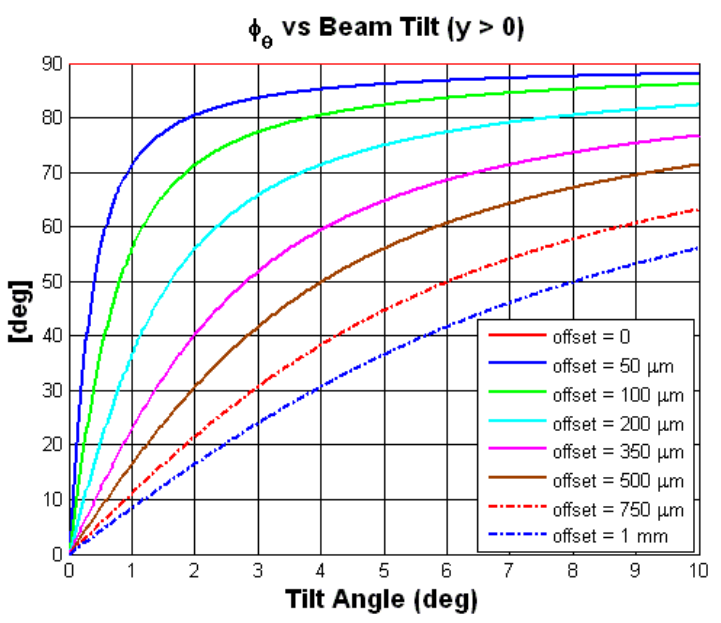

Fig. 5. Equivalent beam-loading current phase $\phi_{\theta}$ vs. beam tilt for various beam offsets.

The expected beam tilt in the last cavity of the first group and the first cavity of the second group for 0.5MV transverse voltage per SPX cavity is $0.004 \mathrm{rad}(0.23 \mathrm{deg})$ in a 4-cavity-per-sector case and $0.017 \mathrm{rad}(0.97 \mathrm{deg})$ in a 8cavity-per-sector case for SPX [9] assuming a cavity-tocavity spacing of $0.15 \mathrm{~m}$. 


\section{Phasor Diagram}

Assuming that electrons at the head of the bunch (arriving early) are kicked up and those at the tail of the bunch (arriving late) are kicked down in the first group of crab cavities of the short-pulse $\mathrm{x}$-ray scheme [10], the electron beam crosses the first cavity group at the negative-sloped zero-crossing of a positive vertical kick, $y^{\prime}(t)$. For electrons, this implies a positive real $V_{t}$ and negative real $V_{m}$ according to the convention of Eqs. 2 to 5 . In the second group of crab cavities, which is intended to cancel the kick imparted by the first cavity group, the electron beam nominally crosses the cavity gap at the positivesloped zero-crossing. This implies a negative real $V_{t}$ and a positive real $V_{m}$.

For the first group of cavities, the time domain transverse kick is equal to a negative sinusoid according to $y^{\prime}(t)=\operatorname{Re}\left\{j \frac{-q V_{t}}{E} \cdot e^{+j \omega t}\right\}=\frac{-e V_{t}}{E} \sin \omega_{o} t \quad$ with $e$ the electron charge. The corresponding time domain longitudinal voltage, denoted as $v_{z}(t)$, is equal to $v_{z}(t)=\operatorname{Re}\left\{V_{m} y \cdot e^{+j \omega t}\right\}=-V_{m} y \cos \omega_{o} t$ as depicted in Fig. 6.

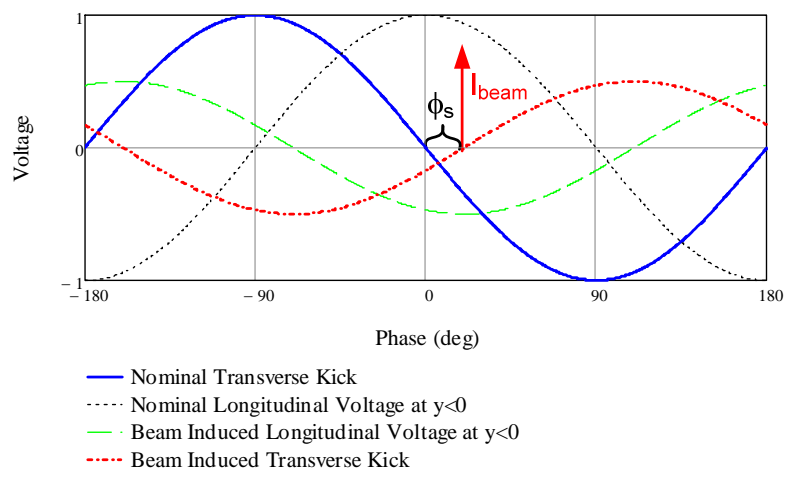

Fig. 6. Depiction of the nominal and beam-induced voltages for the first group of crab cavities with a $+y$ beam offset and no tilt.

The synchronous phase angle, $\phi_{s}$, is defined as the phase of the beam arrival relative to the negative-sloped zerocrossing of $y^{\prime}(t)$. If the beam arrives late, $\phi_{s}>0$. If the beam crosses with a positive vertical offset and no tilt, then the beam loading current $\hat{I}_{B}$ will have a phase angle of $-\left(\pi+\phi_{s}\right)$. For a nominal $\phi_{s}=0$, this implies that the beam-loading voltage for a beam with a pure $y>0$ offset is 180deg out of phase with the desired operating voltage of the first group of cavities and hence receives energy from the generator through the cavity longitudinal electric fields. If the beam has a pure $y<0$ offset, the beam loading is in-phase with the operating voltage of the first group of cavities and is helping to drive the cavity to the intended operating voltage. The converse is true for the second group of cavities. Thus, the beam loading from pure offset is nominally either in-phase (0deg) or out-ofphase (180deg) depending upon beam offset polarity and operating voltage polarity. For practical purposes the four possibilities from the combination of two polarities can be collapsed to two cases for a beam with pure offset: (a) $V_{t} \cdot y>0$ and (b) $V_{t} \cdot y<0$ as depicted in Fig. 7. Note that this beam loading for crabbing cavities is in contrast to deflecting cavities for which the beam loading for pure offset is nominally in quadrature to the intended operating voltage.

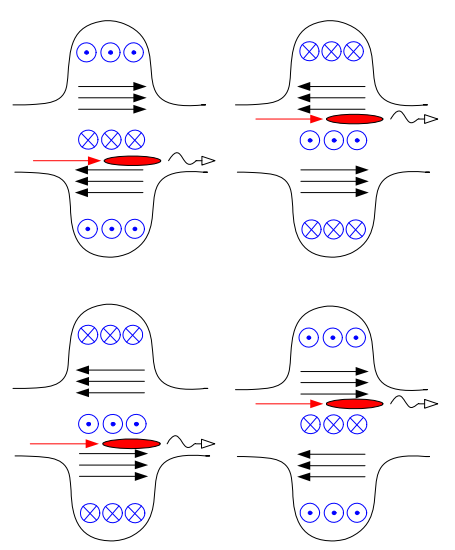

Fig. 7. Electron beam-loading cases for pure offset (a) $V_{t} \cdot y>0$ [top] and (b) $V_{t} \cdot y<0$ [bottom].

The phasor diagram that can be used to describe the beam-loaded equivalent circuit for crab cavities is shown in Fig. 8.

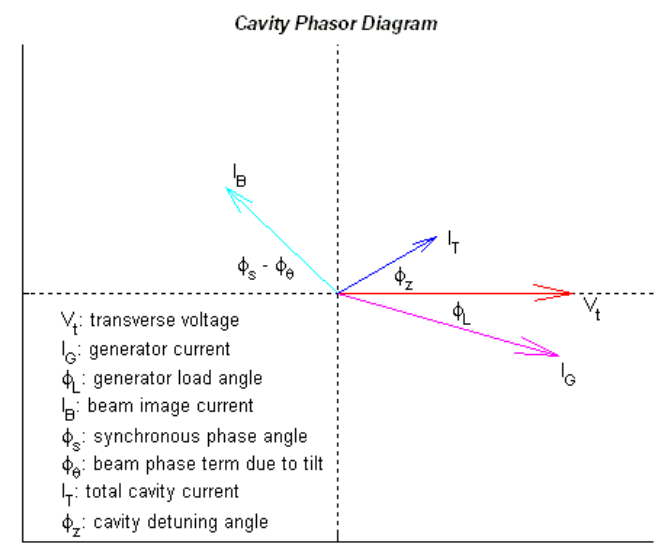

Fig. 8. Cavity phasor diagram for $V_{t} \cdot y>0$.

The steady-state phasors are defined in accordance with the following relationships:

$$
\begin{aligned}
& \hat{I}_{T}=\hat{I}_{G}+\hat{I}_{B} \quad \text { and } \\
& \hat{V}_{t}=\hat{I}_{T} \cdot\left|\hat{Z}_{e q}\right| e^{j \phi_{z}},
\end{aligned}
$$

where $\hat{Z}_{e q}=\frac{R /(\beta+1)}{1-j \tan \phi_{Z}} \quad$ represents the parallel combination of the cavity RLC circuit with input loading, $\hat{I}_{T}$ is the total current (generator + beam-loading) driving the circuit, $\hat{I}_{G}=\left|\hat{I}_{G}\right| e^{j \phi_{L}}$ is the generator current phasor with load angle $\phi_{L}$, and $\hat{I}_{B}$ is the beam-loading current phasor given by Eq. 20 . 


\section{MINIMUM REQUIRED RF POWER}

The steady-state required forward power, $P_{g}^{+}$, from an rf generator needed to produce a desired transverse voltage magnitude, $V_{t}$, in the presence of beam loading and cavity detuning is given as (see Appendix B)

$$
P_{g}^{+}=\frac{V_{t}^{2}}{8 \beta\left(\frac{R}{Q}\right)^{\prime} Q_{o}} \cdot\left[\left(\beta+1+\frac{P_{B}}{P_{c a v}}\right)^{2}+\left((\beta+1) \tan \phi_{Z}+\frac{V_{t}\left|\hat{I}_{B}\right| \sin \phi_{B}}{2 P_{c a v}}\right)^{2}\right]
$$

where $\beta=\frac{Q_{o}}{Q_{e x t}}$ is the input coupling coefficient. $\frac{P_{B}}{P_{\text {cav }}}$ represents the ratio of the power delivered to the beam, $P_{B}$, to the power dissipated in the cavity walls, $P_{c a v}$, where

$$
P_{B}=-\frac{1}{2} V_{t}\left|\hat{I}_{B}\right| \cos \phi_{B}
$$

and $P_{c a v}$ is given by Eq. 7 .

The loaded cavity impedance/(detuning) angle, $\phi_{Z}$, can be approximated by

$$
\tan \phi_{Z} \cong 2 Q_{L} \frac{\omega_{r}+\delta \omega_{M}-\omega_{o}}{\omega_{r}}=2 \frac{Q_{L}}{\omega_{r}}\left(\Delta \omega+\delta \omega_{M}\right),
$$

where $\Delta \omega \equiv \omega_{r}-\omega_{o}$ is defined as the nominal cavity detuning with $\omega_{r}$ the nominal cavity resonant frequency and $\omega_{o}$ the rf drive frequency. Random or uncontrollable fluctuations of the cavity resonant frequency (i.e., from microphonics) are represented by $\delta \omega_{m}$. For a given cavity design, operating gradient, beam current, and nominal synchronous phase angle the free design parameters are the input coupling coefficient, $\beta$, and the cavity detuning, $\Delta \omega$. Thus the right-most term of Eq. 29 can be written as:

$$
\left((\beta+1) \cdot \frac{2 Q_{L}}{\omega_{r}}\left(\Delta \omega+\delta \omega_{m}\right)+\frac{V_{t}\left|\hat{I}_{B}\right| \sin \phi_{B}}{2 P_{c a v}}\right)^{2} .
$$

Assuming that only a slow cavity tuner is available, such that $\Delta \omega$ cannot compensate for the fast uncontrollable fluctuations $\delta \omega_{m}$, then Eq. 32 can only be minimized to

$$
\left((\beta+1) \cdot 2 Q_{L} \frac{\delta \omega_{m}}{\omega_{r}}\right)^{2}=\left(2 Q_{o} \frac{\delta \omega_{m}}{\omega_{r}}\right)^{2}
$$

for an optimum nominal cavity detuning given by

$$
\Delta \omega_{\text {opt }}=-\frac{\omega_{r}}{2 Q_{L}} \cdot \frac{V_{t}\left|\hat{I}_{B}\right| \sin \phi_{B}}{2 P_{c a v}(\beta+1)} .
$$

Thus, at optimal detuning, $\Delta \omega=\Delta \omega_{\text {opt }}$,

$$
\left.P_{g}^{+}\right|_{\Delta \omega_{\text {opt }}}=\frac{V_{t}^{2}}{8 \beta\left(\frac{R}{Q}\right)^{\prime} Q_{o}} \cdot\left[\left(\beta+1+\frac{P_{B}}{P_{c a v}}\right)^{2}+\left(2 Q_{o} \frac{\delta \omega_{m}}{\omega_{o}}\right)^{2}\right]
$$

Minimizing with respect to $\beta$ results in an optimal coupling coefficient of

$$
\beta_{\text {opt }}=\sqrt{\left(1+\frac{P_{B}}{P_{\text {cav }}}\right)^{2}+\left(2 Q_{o} \frac{\delta \omega_{m}}{\omega_{r}}\right)^{2}} .
$$

The minimum steady-state required rf generator power for a single SPX cavity as a function of $\mathrm{Q}_{\text {ext }}$ for various beam offsets assuming no beam tilt and no microphonic detuning is shown in Figs. 9 and 10 for $Q_{o}=1 \cdot 10^{9}$ and $(R / Q)^{\prime}=17.8 \Omega$ [11], $\phi_{s}=0, V_{t}=0.5 \mathrm{MV}$ per cavity, and $\sigma_{t} \cong 40 \mathrm{ps}$ corresponding to an rf component of beam current of $i_{o} \cong 1.56 \cdot I_{D C}$ with $I_{D C}=200 \mathrm{~mA}$.

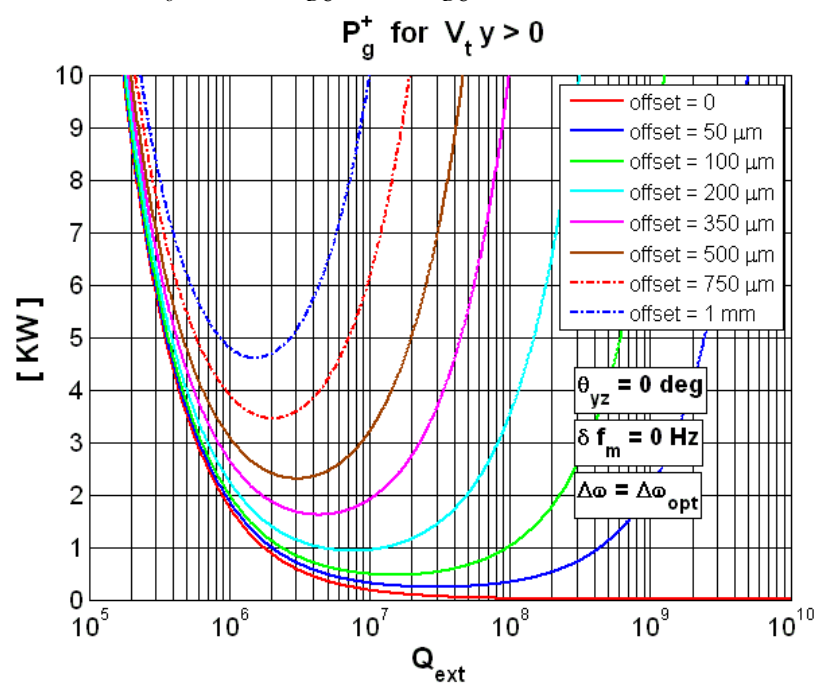

Fig. 9. $P_{g}^{+}$vs. $Q_{e x t}$ for $V_{t} \cdot y>0, \theta_{y z}=0, \phi_{s}=0$, $\delta f_{m}=0$, and $\Delta \omega=\Delta \omega_{o p t}=0$.

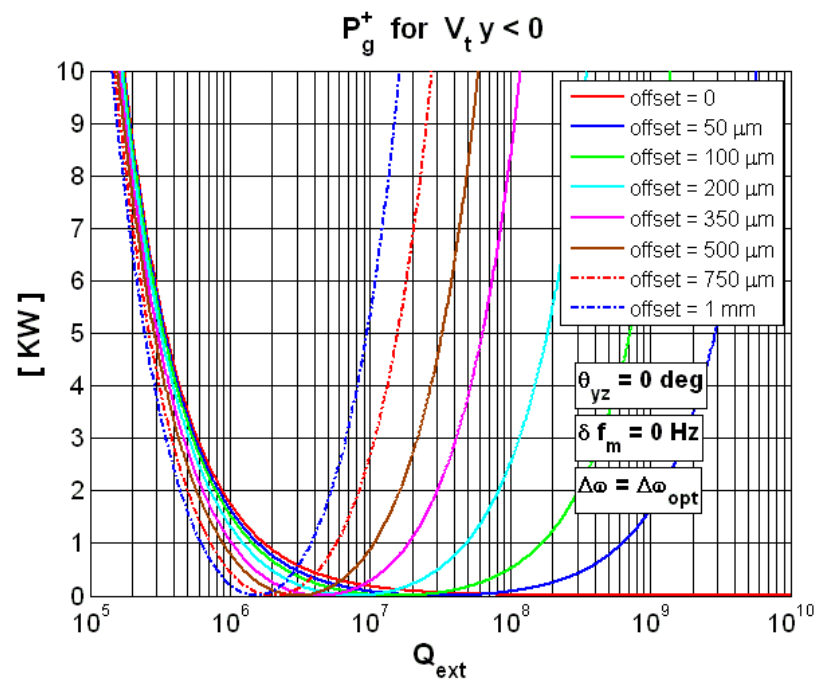

Fig. 10. $P_{g}^{+}$vs. Q Q ${ }_{\text {ext }}$ for $V_{t} \cdot y<0, \theta_{y z}=0, \phi_{s}=0$, $\delta f_{m}=0$, and $\Delta \omega=\Delta \omega_{\text {opt }}=0$. 
Until the microphonic detuning levels can be measured in the final installation environment, an expected value of microphonics may be assumed to be $\sim 17 \mathrm{~Hz}$ rms [11]. This corresponds to a 6-sigma peak level of microphonics of $\sim 100 \mathrm{~Hz}$. The required power for twice this level has been calculated for the same conditions as Figs. 9 and 10 and is shown in Figs. 11 and 12.

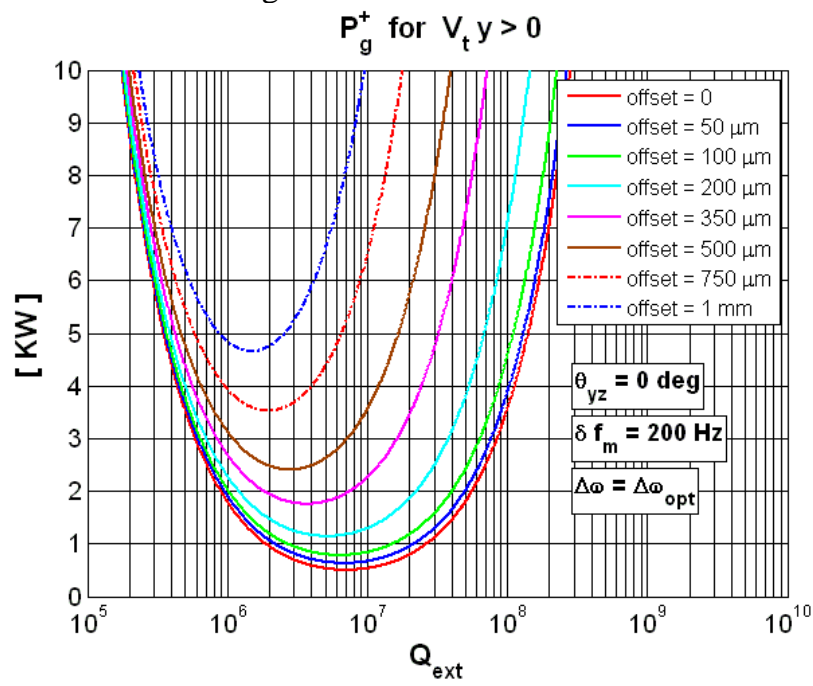

Fig. 11. $P_{g}^{+}$vs. $\mathrm{Q}_{\mathrm{ext}}$ for $V_{t} \cdot y>0, \theta_{y z}=0, \phi_{s}=0$, $\delta f_{m}=200 \mathrm{~Hz}$, and $\Delta \omega=\Delta \omega_{\text {opt }}=0$.

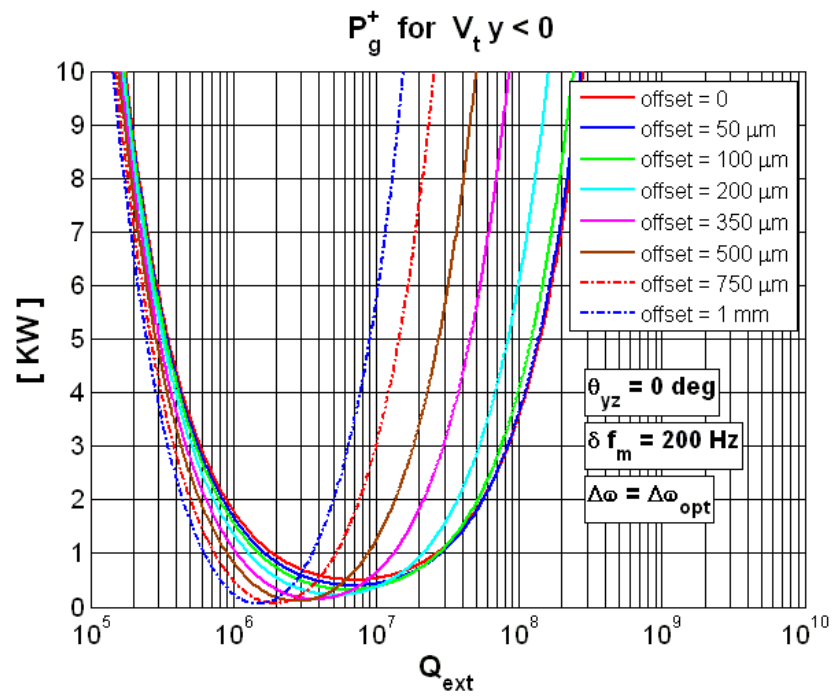

Fig. 12. $P_{g}^{+}$vs. Q Qext for $V_{t} \cdot y<0, \theta_{y z}=0, \phi_{s}=0$, $\delta f_{m}=200 \mathrm{~Hz}$, and $\Delta \omega=\Delta \omega_{\text {opt }}=0$.
To account for beam tilt, the required power was calculated for unfavorable conditions of a 2deg beam tilt with no static detuning $(\Delta \omega=0)$ and assuming that the peak microphonic detuning is in a direction that increases the term represented by Eq. 32. The results are shown in Figs. 13 and 14.

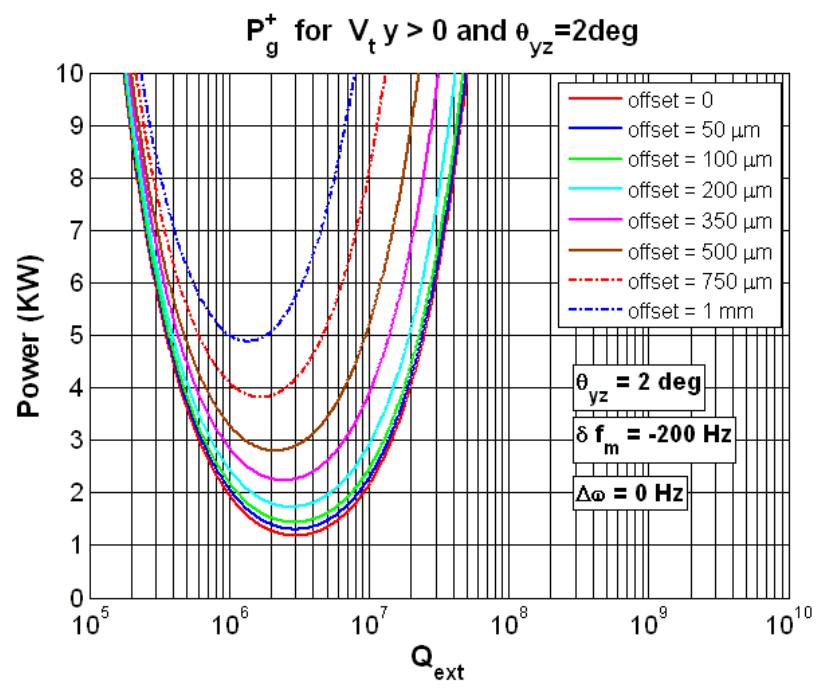

Fig. 13. $P_{g}^{+}$vs. Q Q ext for $V_{t} \cdot y>0, \theta_{y z}=2 \mathrm{deg}, \phi_{s}=0$, $\delta f_{m}=-200 \mathrm{~Hz}$, and $\Delta \omega=0$.

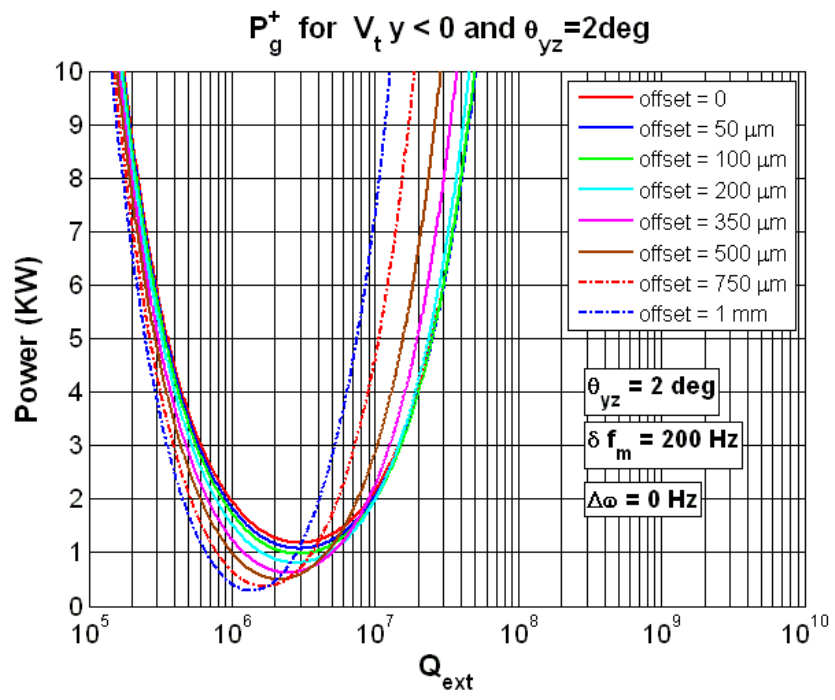

Fig. 14. $P_{g}^{+}$vs. $Q_{e x t}$ for $V_{t} \cdot y<0, \theta_{y z}=2 \operatorname{deg}, \phi_{s}=0$, $\delta f_{m}=+200 \mathrm{~Hz}$, and $\Delta \omega=0$. 


\section{REFLECTED POWER}

The power reflected back to the source/circulator for a single cavity can be determined from (see Appendix B)

$$
P_{g}^{-}=\frac{1}{2} \frac{\left|V_{G}\right|^{2}}{\left(\frac{R}{Q}\right)^{\prime} Q_{e x t}},
$$

where $\hat{V}_{G}^{-}$represents the reflected voltage at the input coupler referenced to the cavity gap and is given in terms of the required generator current and cavity voltage as

$$
\hat{V}_{G}{ }^{-}=\hat{V}_{t}-\frac{1}{2} \hat{I}_{G} \cdot\left(\frac{R}{Q}\right)^{\prime} Q_{e x t} .
$$

The steady-state reflected power was determined for the scenarios used in the previous section to calculate the forward power. The results are shown in Figs. 15-18.

$$
P_{g}^{-} \text {for } V_{t} y>0
$$

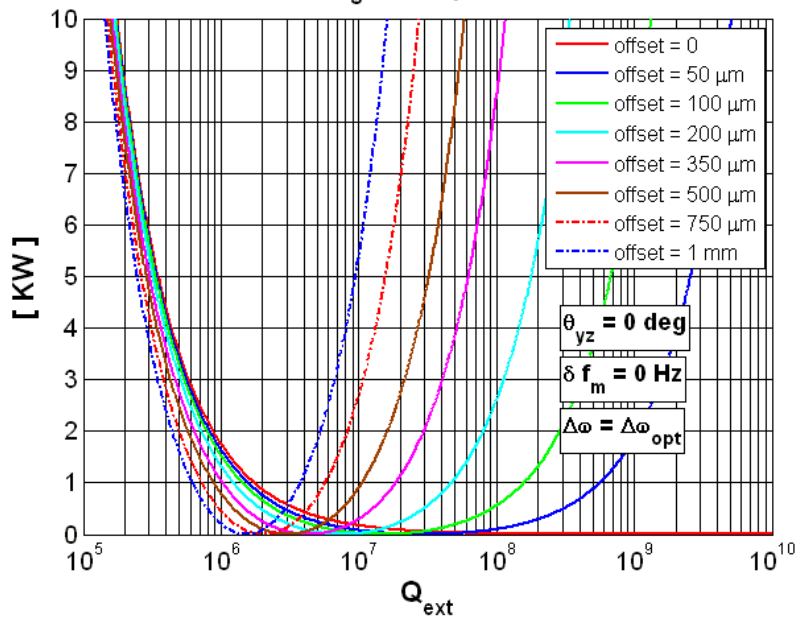

Fig. 15. $P_{g}^{-}$vs. $Q_{e x t}$ for $V_{t} \cdot y>0, \theta_{y z}=0, \phi_{s}=0$, $\delta f_{m}=0$, and $\Delta \omega=\Delta \omega_{\text {opt }}=0$.

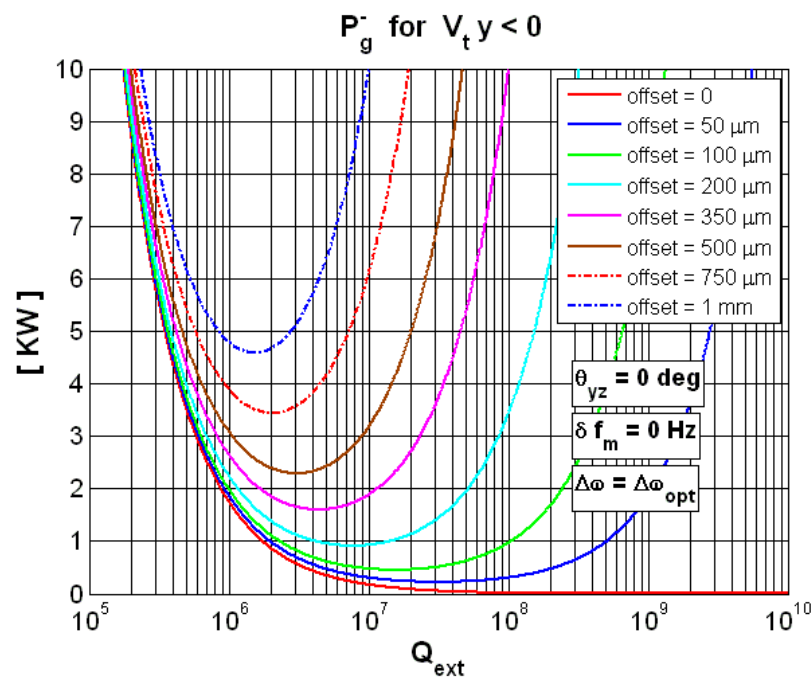

Fig. 16. $P_{g}^{-}$vs. $Q_{e x t}$ for $V_{t} \cdot y<0, \theta_{y z}=0, \phi_{s}=0$, $\delta f_{m}=0$, and $\Delta \omega=\Delta \omega_{\text {opt }}=0$.
In the cases of a negative beam offset the reflected power at optimal coupling for zero detuning is due to the beam driving the cavity and delivering power to the source/circulator. Furthermore, since the cavity wall losses are only $\sim 7 \mathrm{~W}$, the reflected power for $V_{t} \cdot y>0$ is nearly identical to the forward power for $V_{t} \cdot y<0$ and vice versa.

It is important to remember that the reflected power calculations are for steady-state conditions. During transients the peak powers can be higher. In the highly over-coupled superconducting case, at RF turn-off with no beam-loading the peak reflected power can approach 4 times the forward power (see [12] although it was written for the pulsed normal conducting SPX approach).

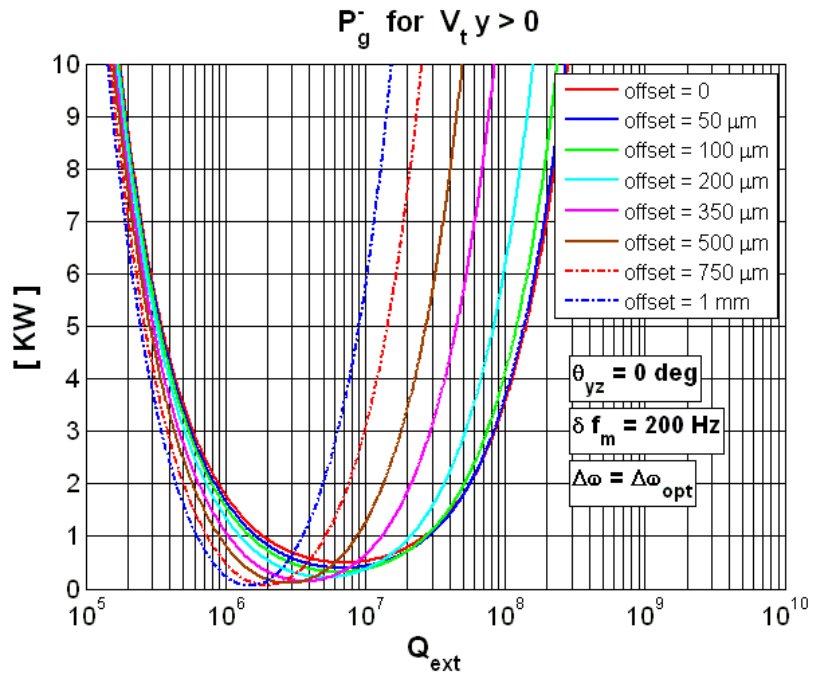

Fig. 17. $P_{g}^{-}$vs. $Q_{e x t}$ for $V_{t} \cdot y>0, \theta_{y z}=0, \phi_{s}=0$, $\delta f_{m}=200 \mathrm{~Hz}$, and $\Delta \omega=\Delta \omega_{\text {opt }}=0$.

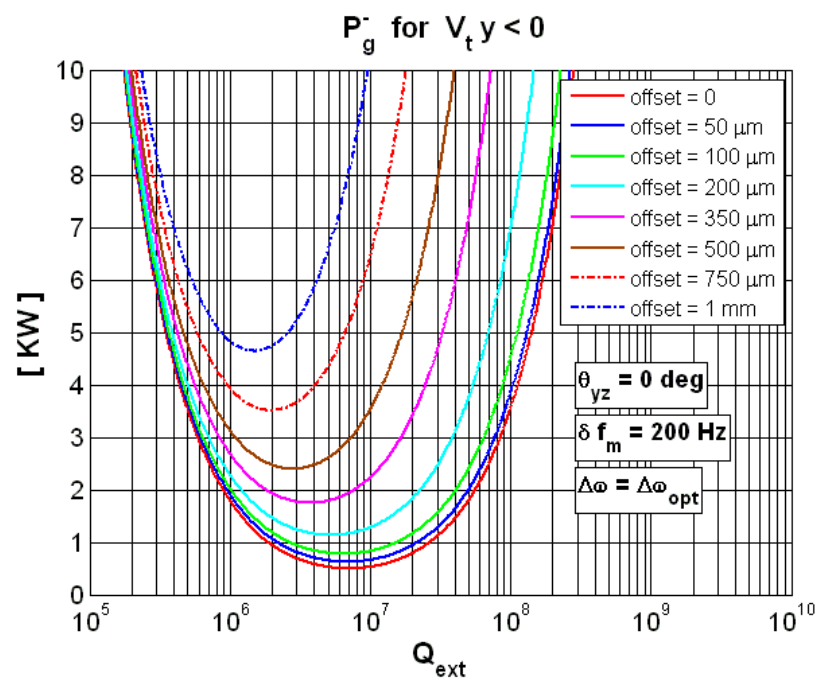

Fig. 18. $P_{g}^{-}$vs. $Q_{e x t}$ for $V_{t} \cdot y<0, \theta_{y z}=0, \phi_{s}=0$, $\delta f_{m}=200 \mathrm{~Hz}$, and $\Delta \omega=\Delta \omega_{\text {opt }}=0$. 


\section{MACHINE PROTECTION CONSIDERATIONS}

For machine protection considerations, the beamgenerated cavity voltage was calculated for pure beam offsets with zero cavity detuning as a function of $Q_{e x t}$ as shown in Fig. 19. The beam-induced reflected power with no rf drive is shown in Fig. 20. Finally, the achievable cavity voltage as a function of $Q_{e x t}$ with zero cavity detuning for various drive power levels is shown in Fig. 21.

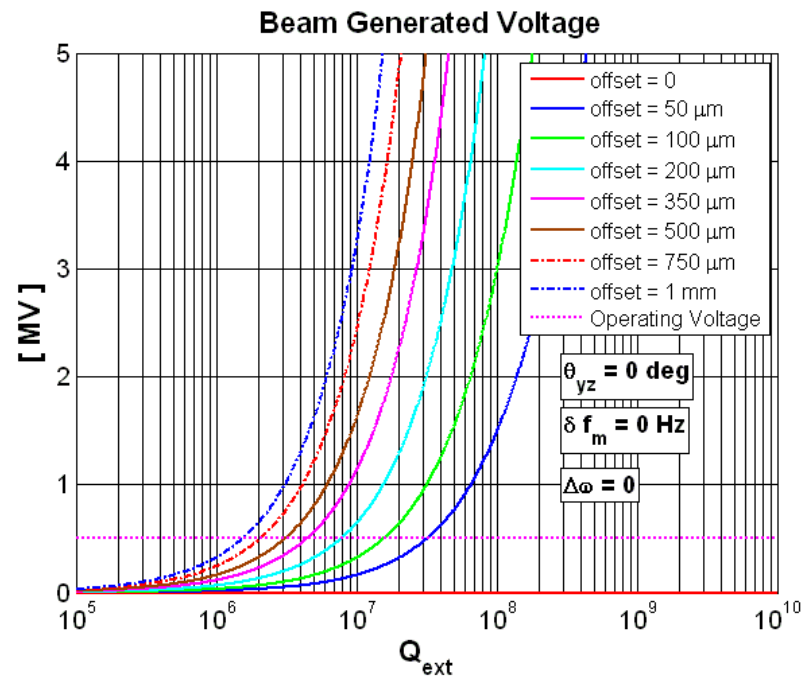

Fig. 19. Beam generated voltage for pure beam offsets vs. $Q_{e x t}$ with $\delta \omega_{m}=0$ and $\Delta \omega=0$.

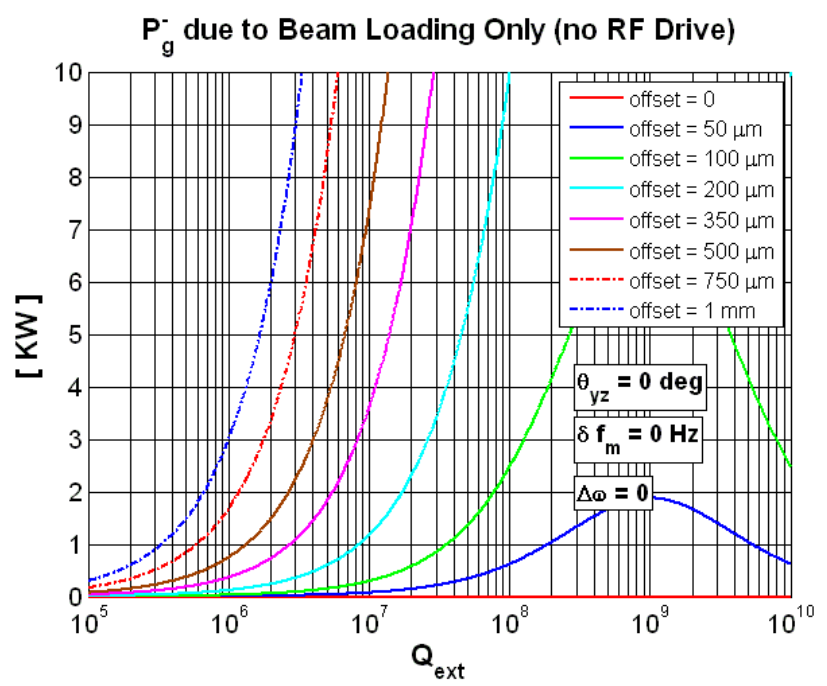

Fig. 20. Beam induced reflected power (no RF drive) for pure beam offsets vs. $Q_{e x t}$ with $\delta \omega_{m}=0$ and $\Delta \omega=0$.

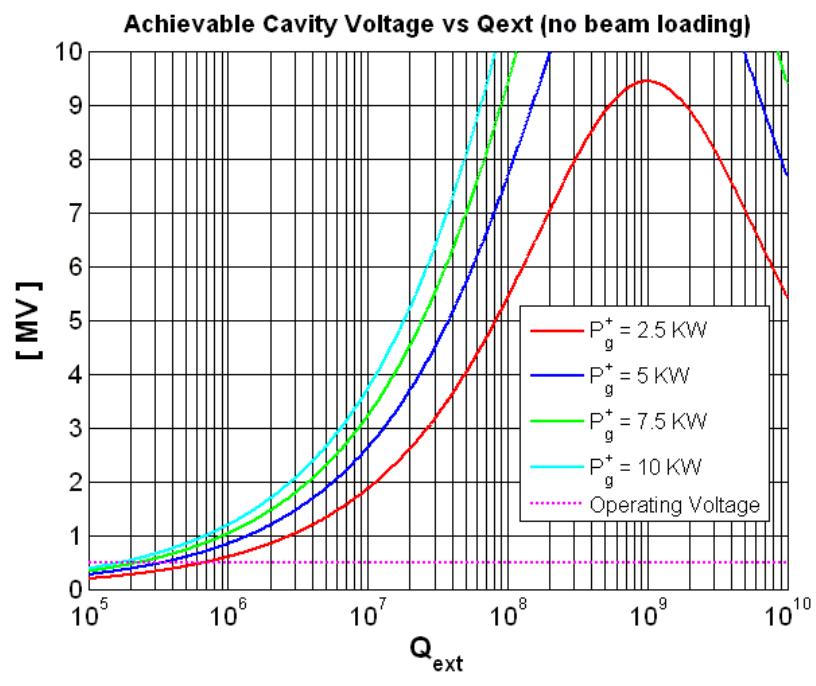

Fig. 21. Achievable cavity voltage vs $Q_{e x t}$ with zero cavity detuning for various drive power levels.

\section{CONCLUSION}

A beam-loaded circuit model for polarized $\mathrm{TM}_{110}$ mode crab cavities was derived. The single-cavity minimum steady-state required generator power has been determined for the APS SPX crab cavities for a storage ring current of $200 \mathrm{~mA}$ DC current as a function of external $Q$ for various vertical offsets including beam tilt and uncontrollable detuning. Calculations to aid machine protection considerations were given.

\section{REFERENCES}

[1] M. McAshan, R. Wanzenberg, "RF Design of a Transverse Mode Cavity for Kaon Separation," Fermilab Technical Memo TM-2144, May 2001. http://lss.fnal.gov/archive/test-tm/2000/fermilab-tm-2144.pdf

[2] L. Emery, "Shunt Impedance Conventions for Power Calculations and for Instability Calculations, version 2,” APS Internal Note, OAG-TN-2007-022, 5/19/2010.

[3] J.C. Slater, “Microwave Electronics,” D.Van Nostrand Company, Inc., New York, 1950.

[4] E.L. Ginzton, "Microwave Measurements," McGrawHill Book Co., New York, 1957, Library of Congress Catalog Number 56-13393, pp. 391-397.

[5] P.B.Wilson, "High Energy Electron Linacs: Applications to Storage Ring RF Systems and Linear Colliders," SLAC-PUB-2884, Nov. 1991.

[6] M. Ross, J. Frisch, D. McCormick, "RF Cavity BPM's as Beam Angle and Beam Correlation Monitors," Proceedings of PAC 2003, pp. 2548-2550.

[7] G. Decker, "Conceptual Design for a Rectangular Cavity BPM / Tilt Monitor for the APS Storage Ring”, APS Internal Note, DIAG-TN-2010-10, Sept. 21, 2010.

[8] D. Boussard, "Beam Loading," Proceedings of the CERN Accelerator School Fifth Advanced Accelerator Physics Course, CERN 95-06(1) pp.415436. 
[9] Vadim Sajaev, personal correspondence

[10] A. Zholents, P. Heimann, M. Zolotorev, \& J. Byrd "Generation of subpicosecond X-ray pulses using RF orbit deflection," Nuclear Instruments and Methods in Physics Research A, 425 (1999) pp. 385-389.

[11] G. Waldschmidt, personal correspondence

[12] T. Berenc, "Klystron Phase Reversal Considerations for SPX,” APS Internal Note, ICMS\# APS_1230024, 11/11/2007.

[13] L. Palumbo, V.G. Vaccaro, M. Zobov, "Wake Fields and Impedance," Proceedings of the CERN Accelerator School Fifth Advanced Accelerator Physics Course, CERN 95-06(1) pp.331-390.

[14] A. Hoffman, "Beam Instabilities," Proceedings of the CERN Accelerator School Fifth Advanced Accelerator Physics Course, CERN 95-06(1) pp.307326. 


\section{APPENDIX A}

As in Ref. [7], a differential charge element for a vertically tilted bunch can be written as:

$$
d q(y, t)=\frac{q}{\sqrt{2 \pi} \sigma_{t}} e^{\frac{-\left(t-t_{o}\right)^{2}}{2 \sigma_{t}^{2}}} \delta\left(y+\theta_{y z} c\left(t-t_{o}\right)-y_{o}\right) d y d t
$$

where $\delta\left(y+\theta_{y z} c\left(t-t_{o}\right)-y_{o}\right)$ is the Dirac delta function, $q$ is the total charge of the bunch, $\sigma_{t}$ is the rms bunch length, $\theta_{y z}$ is the bunch tilt in the $y-z$ plane, $t_{o}$ is the time at which the bunch centroid crosses the cavity gap, $y_{o}$ is the vertical offset of the centroid, and $c$ is the speed of light.

Invoking differential superposition and integrating over the charge distribution as in Ref. [5, Sect. 2.3] gives the following integral for the voltage induced by the entire bunch:

$$
\begin{gathered}
V_{t B 0}=\int \frac{V_{t b 0}(y)}{q} e^{-j \omega_{o} t} d q=\omega_{r}\left(\frac{R}{Q}\right)^{\prime} \kappa_{o} \cdot \int_{-\infty}^{\infty} \int_{-\infty}^{\infty} y \frac{q}{\sqrt{2 \pi} \sigma_{t}} e^{-j \omega_{o} t} e^{\frac{-\left(t-t_{o}\right)^{2}}{2 \sigma_{t}^{2}}} \delta\left(y+\theta_{y z} c\left(t-t_{o}\right)-y_{o}\right) d y d t \\
=\omega_{r}\left(\frac{R}{Q}\right)^{\prime} \kappa_{o} \cdot \int_{-\infty}^{\infty}\left(y_{o}-\theta_{y z} c\left(t-t_{o}\right)\right) \frac{q}{\sqrt{2 \pi} \sigma_{t}} e^{-j \omega_{o} t} e^{\frac{-\left(t-t_{o}\right)^{2}}{2 \sigma_{t}^{2}}} d t \\
=\omega_{r}\left(\frac{R}{Q}\right)^{\prime} \kappa_{o} \cdot\left[\int_{-\infty}^{\infty} y_{o} \frac{q}{\sqrt{2 \pi} \sigma_{t}} e^{-j \omega_{o} t} e^{\frac{-\left(t-t_{o}\right)^{2}}{2 \sigma_{t}^{2}}} d t-\int_{-\infty}^{\infty} \theta_{y z} c\left(t-t_{o}\right) \frac{q}{\sqrt{2 \pi} \sigma_{t}} e^{-j \omega_{o} t} e^{\frac{-\left(t-t_{o}\right)^{2}}{2 \sigma_{t}^{2}}} d t\right] \text { (A2), }
\end{gathered}
$$

where $V_{t b 0}(y)=q \cdot \omega_{r}\left(\frac{R}{Q}\right)^{\prime} \kappa_{o} y$ is the point charge induced transverse voltage as derived in the main text.

The first integral of Eq. A2 can be recognized to have the form of a Fourier transform integral of a time delayed Gaussian. Using the Fourier transform, F, of a Gaussian

$$
F\left\{\frac{1}{\sqrt{2 \pi} \sigma_{t}} e^{\frac{-t^{2}}{2 \sigma_{t}^{2}}}\right\}=e^{\frac{-\left(\omega_{o} \sigma_{t}\right)^{2}}{2}} \text { and the time delay property of Fourier transforms } F\left\{f\left(t-t_{o}\right)\right\}=e^{-j \omega_{o} t_{o}} F\{f(t)\}
$$

the first integral is found to be

$$
\int_{-\infty}^{\infty} y_{o} \frac{q}{\sqrt{2 \pi} \sigma_{t}} e^{-j \omega_{o} t} e^{\frac{-\left(t-t_{0}\right)^{2}}{2 \sigma_{t}^{2}}} d t=q y_{o} e^{-j \omega_{o} t_{o}} e^{\frac{-\left(\omega_{o} \sigma_{t}\right)^{2}}{2}}
$$

The second integral of Eq. A2 can be recognized as the Fourier transform integral of a Gaussian multiplied by t. To account for the multiplication by t, the frequency differentiation property of the Fourier transform can be invoked which is given as:

$$
\text { Frequency Differentiation Property of the Fourier Transform: } F\{t f(t)\}=j \frac{d}{d \omega} F\{f(t)\}
$$

Thus, the Fourier transform integral of a Gaussian multiplied by $\mathrm{t}$ is given as:

$$
F\left\{t \cdot \frac{1}{\sqrt{2 \pi} \sigma_{t}} e^{\frac{-t^{2}}{2 \sigma_{t}^{2}}}\right\}=-j \omega_{o} \sigma_{t}^{2} e^{\frac{-\left(\omega_{o} \sigma_{t}\right)^{2}}{2}}
$$

By additionally using the time delay property of the Fourier transform, the second integral of Eq. A2 becomes:

$$
-\int_{-\infty}^{\infty} \theta_{y z} c\left(t-t_{o}\right) \frac{q}{\sqrt{2 \pi} \sigma_{t}} e^{-j \omega_{o} t} e^{\frac{-\left(t-t_{o}\right)^{2}}{2 \sigma_{t}^{2}}} d t=j q \theta_{y z} \omega_{o} c \sigma_{t}^{2} e^{-j \omega_{o} t_{o}} e^{\frac{-\left(\omega_{o} \sigma_{t}\right)^{2}}{2}}
$$

Combining A2 through A4, the transverse voltage induced by a tilted bunch is given as:

$$
V_{t B 0}=q \cdot e^{\frac{-\left(\omega_{o} \sigma_{t}\right)^{2}}{2}} \kappa_{o} \omega_{r}\left(\frac{R}{Q}\right)^{\prime}\left[y_{o}+j \theta_{y z} \omega_{o} c \sigma_{t}^{2}\right] \cdot e^{-j \omega_{o} t_{o}}
$$




\section{APPENDIX B}

A cavity driven by a klystron with a circulator can be represented by the circuit of Fig. B1, where the cavity is modeled as a lumped RLC circuit and the input coupler is modeled as a transformer [3-4] with turns ratio $\mathrm{N}$.

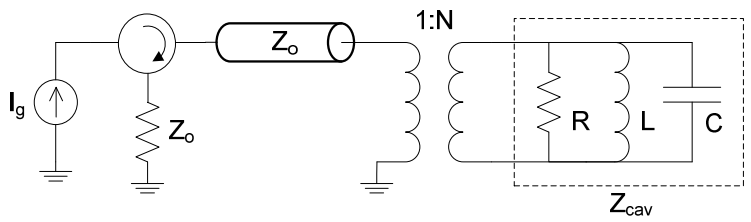

Fig. B1. Circuit model of a cavity driven by a klystron with a circulator.

The klystron source, $I_{g}$, with a circulator can be shown to be equivalent to a current source of magnitude $2 I_{g}$ with source impedance $Z_{o}$. Both models result in the same forward voltage to the cavity $V_{g}^{+}=I_{g} Z_{o}$. Thus, the circuit of Fig. 19 can be replaced with the circuit of Fig. B2.

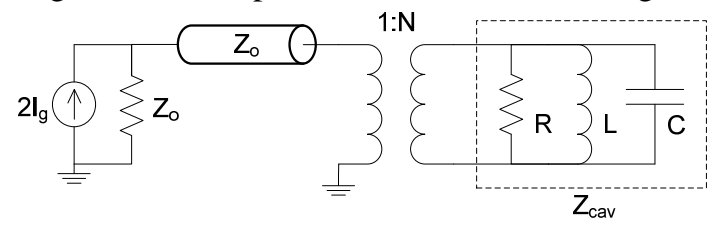

Fig. B2. Equivalent circuit with klystron and circulator replaced by a current source with source impedance $Z_{o}$.

The cavity impedance can be expressed as $Z(\omega)=\frac{R}{1-j \tan \phi_{o}}$, where $R$ is the cavity shunt impedance and $\phi_{o}$ is the unloaded cavity detuning angle given by $\tan \phi_{o}=Q_{o} \frac{\omega_{r}^{2}-\omega_{o}^{2}}{\omega_{r} \omega_{o}} \cong 2 Q_{o} \frac{\Delta \omega}{\omega_{r}}$, with $Q_{o}$ the cavity unloaded quality factor, $\omega_{r}$ the cavity resonant frequency, $\omega_{o}$ the rf drive frequency, and $\Delta \omega \equiv \omega_{r}-\omega_{o}$. The input coupling coefficient is defined as the ratio of the external losses to the cavity losses, $\beta=\frac{P_{\text {ext }}}{P_{\text {cav }}}=\frac{Q_{o}}{Q_{\text {ext }}}=\frac{R_{S}}{N^{2} Z_{o}}$.

Transforming the generator onto the cavity gap and adding a current source to represent beam loading results in the circuit of Fig. B3.

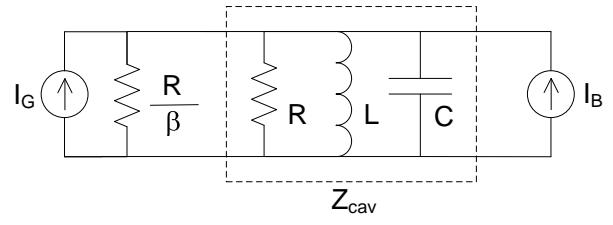

Fig. B3. Equivalent circuit with generator transformed onto the cavity gap and including a beam loading source.

Using the phasor diagram shown in Fig. 8 of the main text, the steady-state equations are

$$
\hat{I}_{T}=\left|\hat{I}_{G}\right| e^{j \phi_{L}}+\left|\hat{I}_{B}\right| e^{j \phi_{B}}
$$

$$
\text { and } \hat{V}_{T}=\hat{I}_{T} \cdot\left|\hat{Z}_{e q}\right| e^{j \phi_{z}} \text {, }
$$

where $\quad \hat{Z}_{e q}=\frac{R /(\beta+1)}{1-j \tan \phi_{Z}} \quad$ represents the parallel combination of the cavity RLC circuit with input loading with loaded detuning angle $\phi_{Z}$ given from $\tan \phi_{Z}=Q_{L} \frac{\omega_{r}^{2}-\omega_{o}^{2}}{\omega_{r} \omega_{o}} \cong 2 Q_{L} \frac{\Delta \omega}{\omega_{r}}$ where $Q_{o}=(\beta+1) Q_{L}$ with $Q_{L}$ the loaded $Q$. For a given deflecting voltage $\hat{V}_{t}=V_{t} e^{j 0}$ Eq. B1 is used to solve for the required generator current $\hat{I}_{G}$ which results in:

$\hat{I}_{G}=\left[\frac{V_{t}(\beta+1)}{R}-\left|\hat{I}_{B}\right| \cos \phi_{B}\right]-j\left[\frac{V_{t}(\beta+1)}{R} \tan \phi_{Z}+\left|\hat{I}_{B}\right| \sin \phi_{B}\right]$

From circuit theory, the forward power from the generator is given as $P_{g}^{+}=\frac{R}{8 \beta}\left|\hat{I}_{G}\right|^{2}$ which, using Eq. B3 can be

written as

$$
P_{g}^{+}=\frac{V_{t}^{2}}{8 \beta\left(\frac{R}{Q}\right)^{\prime} Q_{o}} \cdot\left[\left(\beta+1+\frac{P_{B}}{P_{c a v}}\right)^{2}+\left((\beta+1) \tan \phi_{Z}+\frac{V_{t}\left|\hat{I}_{B}\right| \sin \phi_{B}}{2 P_{c a v}}\right)^{2}\right]
$$

where the cavity parameters $(R / Q)^{\prime}$ and $Q_{o}$ have been used in place of $R$ according to $R=\left(\frac{R}{Q}\right)^{\prime} \cdot Q_{o}$ and $P_{B}$ and $P_{c a v}$ are defined as: $P_{B}=-\frac{1}{2} V_{t}\left|\hat{I}_{B}\right| \cos \phi_{B}$ and ${ }_{P_{c a v}}=\frac{1}{2} \frac{V_{t}^{2}}{\left(\frac{R}{Q}\right)^{\prime} Q_{o}}$.

The power reflected back to the source/circulator can be determined as follows:

$$
\hat{V}_{G}{ }^{+}+\hat{V}_{G}^{-}=\hat{V}_{t}
$$

where $\hat{V}_{G}{ }^{+}$represents the forward voltage from the source on the cavity side of the transformer and $\hat{V}_{G}^{-}$the reflected voltage. From transmission line circuit theory, the forward voltage can be expressed in terms of the required generator current as:

$$
\hat{V}_{G}^{+}=\frac{1}{2} \hat{I}_{G} \cdot \frac{R}{\beta}=\frac{1}{2} \hat{I}_{G} \cdot\left(\frac{R}{Q}\right)^{\prime} Q_{e x t} \cdot
$$

Thus,

$$
\hat{V}_{G}{ }^{-}=\hat{V}_{t}-\frac{1}{2} \hat{I}_{G} \cdot\left(\frac{R}{Q}\right)^{\prime} Q_{e x t}
$$

and the reflected power is given as:

$$
P_{g}^{-}=\frac{1}{2}\left|V_{G}^{-}\right|^{2} /(R / \beta)=\frac{1}{2} \frac{\left|V_{G}^{-}\right|^{2}}{\left(\frac{R}{Q}\right)^{\prime} Q_{e x t}} .
$$

